\title{
Comprehensive modeling and parameter identification of wind farms based on wide-area measurement systems
}

\author{
Yinfeng WANG ${ }^{1}$, Chao LU ${ }^{1}$, Lipeng ZHU ${ }^{1}$, Guoli ZHANG ${ }^{2}$, \\ Xiu LI' ${ }^{2}$, Ying CHEN ${ }^{2}$
}

\begin{abstract}
With intermittence and stochastics of wind power largely introduced into power systems, power system stability analysis and control is in urgent need of reliable wind farm models. Considering the superiority of wide-area measurement systems, this paper develops a novel methodology for practical synchrophasor measurement-based modeling and parameter identification of wind farms. For the sake of preserving basic structural characteristics and control patterns simultaneously, a comprehensive wind farm model is constructed elaborately. To improve the efficiency of the identification procedure, dominant parameters are classified and selected by trajectory sensitivity analysis. Furthermore, an improved genetic
\end{abstract}

CrossCheck date: 16 May 2016

Received: 1 December 2015/Accepted: 6 June 2016/Published online: 20 July 2016

(c) The Author(s) 2016. This article is published with open access at Springerlink.com

$\triangle$ Chao LU

luchao@tsinghua.edu.cn

Yinfeng WANG

thuwyf2010@163.com

Lipeng ZHU

zhulpwhu@126.com

Guoli ZHANG

yiwinkwz@163.com

Xiu LI

1jj579@163.com

Ying CHEN

285512967@qq.com

1 State Key Laboratory of Power System, Department of Electrical Engineering, Tsinghua University, Beijing 100084, China

2 Power Research Institute, Eastern Inner Mongolia Power Grid of China, Saihan District, Hohhot, China algorithm is proposed to strengthen the capability of global optimization. The test results on the WECC benchmark system and the CEPRI 36-bus system demonstrate the effectiveness and reliability of the proposed modeling and identification methodology.

Keywords Wind farm, Trajectory sensitivity, Dominant parameter, Improved genetic algorithm (IGA), Parameter identification

\section{Introduction}

The increasing penetration of wind power into power systems has brought tremendous challenges to system operation and maintenance especially in the sense that power system stability analysis and control seem to be largely dependent on the reliability of wind farm models. If the modeling issue is not treated felicitously, relevant misleading analysis and control measures may improperly hamper the absorption of large scale wind power [1-4]. For the past few decades, several kinds of wind farm models have been established to study those problems such as security and economic operation [5], subsynchronous resonance [6], operational outage [7], etc. The research communities have made various efforts, mainly involving two categories of approaches, to cope with the modeling issue. Some researchers attempt to classify and cluster the wind turbine generators (WTGs) based on some dynamic parameters or response characteristics in a large wind farm [8-10]. However, the effectiveness of those clustering criterions including input wind speed, rotor slip and rotor current are difficult to be theoretically verified. The others make the whole wind farm equivalent to a single wind generator, which focus primarily on the whole wind farm's response behaviors, yet omitting the complex location 
information and electrical connections [11-13]. However, both kinds of approaches attempting to meet the parameter identification requirements establish reduced-order and simplified wind farm models at the cost of deteriorating some control effects and dynamic responses [14, 15].

In addition to the unreliable models, inaccurate model parameters further exacerbate the modeling problem. Generally, the parameters for manufacturer-specific models are regarded as classified commercial data, and not suit for each kinds of models [16]. Nowadays, the empirical parameters are widely used in practice by dispatchers for convenience. What's worse, almost all of the wind farms within a region are usually modelled with the unified parameters, which is clearly not in accordance with the practical scenarios. A survey of a regional power grid in China conducted by the authors shows that almost 91\% of the wind farm models (see Table A1 in Appendix A) are set by the same empirical parameters in power system stability simulation, which further illustrates this ubiquitous problem. Empirical parameters based models, ignoring the practical dynamics of wind farms, may cause potential hazards in power system security and stability analysis. For example, the simulation with a certain set of empirical parameters after power system faults may draw an optimistic conclusion, while probably drawing a pessimistic one with another set. Therefore, instead of adopting empirical parameters, it is imperative to identify the parameters based on field or practical measurements.

With the rapid development of wide-area measurement systems in recent years, phase measurement units (PMUs) and wide-area measurement systems (WAMS) based modeling approaches have been widely used on various occasions to effectively reflect the devices' real dynamic characteristics, such as modeling of generator, excitation system and dynamic load [17-19]. PMUs are capable of providing reliable field measurement data that contains synchronous and dynamic information, which can meet the requirement of respectively modeling and identifying individual wind farms within a region.

In this paper, a novel wind farm modeling approach based on WAMS is proposed to deal with the unreliability and inaccuracy of conventional wind farm modeling methods. Firstly, a dynamic equivalent model of doubly fed induction generator (DFIG) wind farm is elaborately constructed to simulate the overall characteristics of the wind farm for system analysis. Then, dominant parameters are selected by trajectory sensitivity analysis to tackle the intricate and laborious identification issue of multiple parameters. Additionally, a method incorporating model simulation and the improved genetic algorithm (IGA) is employed to search the wind farm's optimal parameter combination based on the PMU data.

\section{Generic model of wind farm}

The equivalent model based on the G.E. standard DFIG model, recommended by Western Electricity Coordinating Council's (WECC) [20], is established in the MATLAB/ Simulink. As shown in Fig. 1, its main components are divided into four modules: two-mass shafting module, pitch angle control module, reactive control module and wind farm interface module. The frequency control and reactive power droop control are omitted.

\section{1) Two-mass shafting module}

Actually, there will be some difference dynamic response characteristics due to the distinction between structure and mass of wind turbine shaft and wind generator shaft. So twomass module is closer to the actual conditions than one mass module. Ignoring the gearbox, two blocks with different inertia coefficients are used to represent the mechanical driving system, which is described as

$$
\left\{\begin{array}{l}
T_{w t} \frac{\mathrm{d} \omega_{w t}}{\mathrm{~d} t}=\frac{P_{m e c h}}{\omega_{w t}}-K_{t g}\left(\theta_{w t}-\theta_{g}\right)-D_{t g}\left(\omega_{w t}-\omega_{g}\right) \\
T_{g} \frac{\mathrm{d} \omega_{g}}{\mathrm{~d} t}=-\frac{P_{\text {elec }}}{\omega_{g}}+K_{t g}\left(\theta_{w t}-\theta_{g}\right)+D_{t g}\left(\omega_{w t}-\omega_{g}\right) \\
\frac{\mathrm{d} \theta_{w t}}{\mathrm{~d} t}=\omega_{w t} \\
\frac{\mathrm{d} \theta_{g}}{\mathrm{~d} t}=\omega_{g}
\end{array}\right.
$$

where $T_{w t}$ and $T_{g}$ represent the inertia coefficients of the equivalent wind turbine and generator, respectively; $\theta_{w t}$ and $\theta_{g}$ are the torsion angles; $\omega_{w t}$ and $\omega_{g}$ stand for the rotational speeds; $P_{\text {mech }}$ and $P_{\text {elec }}$ are mechanical and electromagnetic powers; $K_{t g}$ is stiffness coefficient and $D_{t g}$ is damping coefficient.

\section{2) Pitch angle control module}

The DFIG wind farms always adopt variable-speed constant-frequency (VSCF) control strategy. The pitch angle control module consists of two parts, pitch control and pitch compensation, as shown in Fig. 2. This module comes into

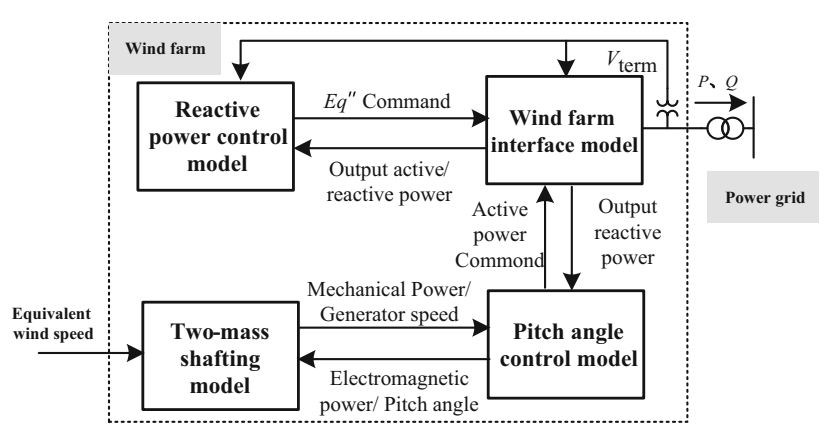

Fig. 1 Schematic diagram of the wind farm module 


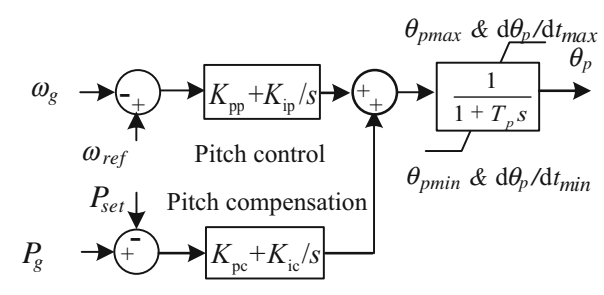

Fig. 2 Pitch angle control module

play, when the wind speed exceeds the rated speed or when the wind farm suffers from serious transient faults.

\section{3) Reactive control module}

The voltage of point of interconnection (POI) and reactive power are governed by closed-loop control strategy in this module. Constant power factor, constant reactive power and constant voltage control modules are optional according to different reactive power reference. The diagram is shown in Fig. A1 in Appendix A.

\section{4) Wind farm interface module}

The whole wind farm is considered as a controllable current source in this module. However, the equivalent generator is not particularly modeled, because the rotor part and excitation system are separately included in the two-mass shafting module and the reactive control module. The impedances of the wind generators and the collection system are all considered in a wind farm's equivalent impedance. The diagram is shown in Fig. 3.

Compared with the conventional reduced-order model in $[14,15]$, the equivalent wind farm model composed of almost all the basic control units is able to better simulate the actual dynamic response, which will be verified by a comparison given in Section 5.

\section{Selection of dominant parameters}

The wind farm model established in the Section 1 preserves the response features to a great extent. However, there are too many parameters needed to be identified. All

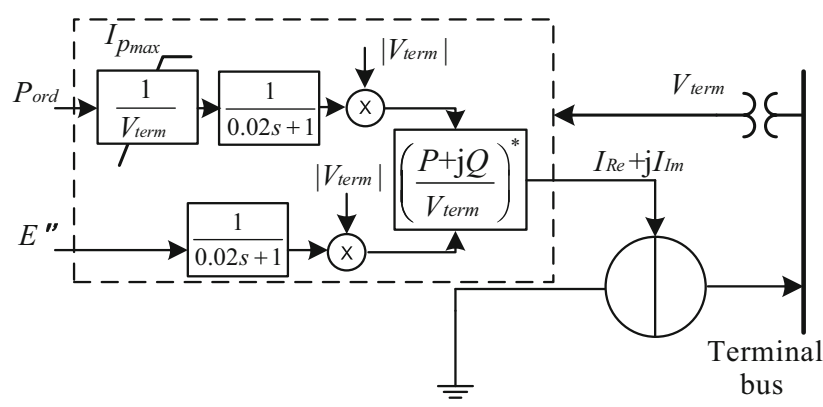

Fig. 3 Wind farm interface module the parameters and their empirical values [20] are listed in Table A2 in Appendix A. It is obvious that not all of them have significantly effects on the model response. Those parameters that dominate more in the model behaviors, namely dominant parameters, should be set accurately.

Identifiability generally means that the model parameters' property of being recognized uniquely based on the model's input and output variables. Most conventional parameter identifiability analysis approaches mainly rely on analytics. They can provide accurate analytical solutions based on linear models,but they are impractical for high-complexity models. Researches in [21, 22] have found that there is inner relationship between the identifiability and sensitivity. Those parameters with high sensitivities are easily to be identified. Hence the sensitivity analysis method is employed in this paper to selected the dominant parameters that easier to be identified as well.

The nonlinear model of wind farm can be expressed as differential-algebraic equations

$\left\{\begin{array}{l}\frac{\mathrm{d}}{\mathrm{d} t} \boldsymbol{x}=f(\boldsymbol{x}, \boldsymbol{y}, \boldsymbol{u}, \boldsymbol{\theta}) \\ \boldsymbol{y}=g(\boldsymbol{x}, \boldsymbol{u}, \boldsymbol{\theta})\end{array}\right.$

where the vector $\boldsymbol{x}$ represents the state variables (such as the $q$-axis subtransient electromotive force $E_{q}^{\prime \prime}$, rotor speed $\omega_{g}$ etc.); vector $\boldsymbol{u}$ is the input variables (such as the wind speed $V_{w}$, the POI bus voltage $V_{\text {term }}$ ); vector $\boldsymbol{\theta}$ stands for the parameters; vector $\boldsymbol{y}$ is the output of the wind farm model.

The parameter sensitivity reflects the output variables' level of sensitivity to the parameter variation. Thus the parameter sensitivity in a certain state is defined as

$S_{y_{i} / \theta_{i}}=\frac{\partial y_{i} / y_{i}}{\partial \theta_{i} / \theta_{i}}=\lim _{\Delta \theta_{i} \rightarrow 0} \frac{\frac{y_{i}\left(\theta_{r}, \theta_{i 0}+\Delta \theta_{i}\right)-y_{i j}\left(\theta_{r}, \theta_{i 0}\right)}{y_{i}\left(\theta_{r}, \theta_{i 0}\right)}}{\Delta \theta_{i} / \theta_{i 0}}$

where $S_{y_{i} / \theta_{i}}$ is the sensitivity of $y_{i}$ related to $\theta_{i} ; y_{i 0}$ is the model output when the $\theta_{i}$ is $\theta_{i 0} ; \Delta \theta_{i}$ is the change of $\theta_{i} ; \theta_{r}$ represents the other parameters except $\theta_{i}$.

The parameter sensitivity can be expressed as an analytical expression, only if the model on an operation point is easy to be linearized. In this paper, a tiny disturbance is added to the parameter to change the wind farm response during a dynamic process. Then the parameter sensitivity is approximatively calculated by (3). Note that the variable $\boldsymbol{y}$ is a function of time, and the parameter sensitivities from different instances form a trajectory sensitivity curve.

Compared with the sensitivity, the trajectory sensitivity with dynamic features added, can be devoted to analyzing the parameters' relation. The parameters whose trajectory sensitivity curves have same phases or contrary phases couple with each other, and cannot be independently identified.

The procedure of selecting dominant parameters is divided into two steps. First, the independent parameters 
are selected by the phase of the trajectory sensitivity. Then, the parameters with larger amplitudes of trajectory sensitivity are treated as the dominant parameters. To make comprehensive comparisons of those parameters' magnitudes of trajectory sensitivity, the average of absolute values of each parameter's trajectory sensitivity in the observed time is computed by

$\bar{S}_{y_{i} / \theta_{i}\left[t_{1}, t_{2}\right]}=\frac{1}{N} \sum_{k=0}^{N}\left|S_{y_{i} / \theta_{i}\left(t_{1}+k \cdot \frac{t_{2}-t_{1}}{N}\right)}\right|$

where $\bar{S}_{y_{i} / \theta_{i}\left[t_{1}, t_{2}\right]}$ is the average sensitivity in the time domain from $t_{1}$ to $t_{2} ; N$ is the number of samples; $S_{y_{i} / \theta_{i}\left(t_{1}+k \cdot \frac{t_{2}-t_{1}}{N}\right)}$ stands for the sensitivity at time instance $t_{1}+k\left(t_{2}-t_{1}\right) / N$.

\section{Parameter identification based on IGA}

\subsection{Identification model}

Essentially, model parameter identification is a procedure of optimization. The goal is to minimize the total square error of the actual output and the predicted output by reasonable adjustment of equivalent parameter under the constraints of state variables and structure parameters.

Because of the extensive application of wide-area measurements, the active and reactive power injection, as well as the voltage at a terminal bus can be obtained credibly. In terms of the identification model here, the active and reactive power injection serve as the output variables, while the terminal voltage and the equivalent wind speed are chosen as the input variables. Note that the equivalent wind speed is calculated with the consideration of wake and tower-shadow effects, which is not the main focus in this paper. There are more state variables than the reduced-order models, owing to the complicated model construction, such as the pitch angle $\theta_{p}$, the generator rotor speed $\omega_{g}$, the wind turbine shaft speed $\omega_{w t}$, and $q$-axis subtransient electromotive force $E_{q}^{\prime \prime}$. The dominant parameters selected for identification will be discussed in Section 5. Now they are represented as the vector $\boldsymbol{\theta}$. The model of parameter identification is given as

$$
\begin{aligned}
& \min \int_{t_{1}}^{t_{2}}\left[|\hat{P}(t)-P(t)|^{2}+|\hat{Q}(t)-Q(t)|^{2}\right] \mathrm{d} t \\
& \text { s.t. }\left\{\begin{array}{l}
\theta_{\text {pmin }} \leq \hat{\theta}_{p} \leq \theta_{\text {pmax }}, E_{q \min }^{\prime \prime} \leq \hat{E}_{q}^{\prime \prime} \leq E_{q \max }^{\prime \prime} \\
\omega_{\text {gmin }} \leq \hat{\omega}_{g} \leq \omega_{\text {gmax }}, \omega_{\text {wtmin }} \leq \hat{\omega}_{w t} \leq \omega_{\text {wtmax }} \\
\boldsymbol{\theta}_{\text {min }} \leq \hat{\boldsymbol{\theta}} \leq \boldsymbol{\theta}_{\text {max }}
\end{array}\right.
\end{aligned}
$$

here the variables with hats mean estimation values. The dominant parameters' boundaries $\boldsymbol{\theta}_{\min }$ and $\boldsymbol{\theta}_{\max }$ are

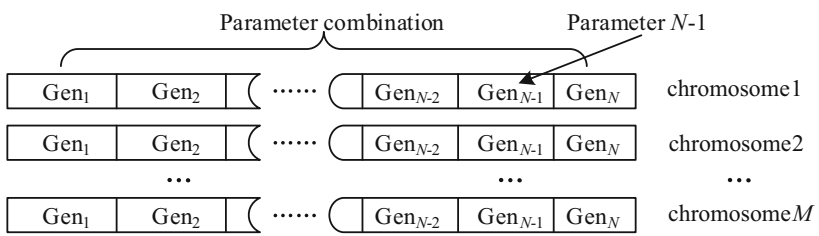

Fig. 4 Representation of parameter combination in GA

determined by their empirical values, so as to guarantee the reasonability of the results.

\subsection{Identification algorithm}

To deal with the problem that the high-order models are too complex to perform efficient parameter identification, the genetic algorithm (GA) that less relies on the internal details of the model is taken into account in this paper. The whole wind farm model is treated as a black box. Only the input and output information is used to search for the optimal parameter combination that matches the dynamic response characteristic. Every parameter combination in GA corresponds to a chromosome, and each parameter in the combination is represented as a gene, as described in Fig. 4. Each parameter to be identified is expressed with a 15-bit binary number, indicating a resolution of 0.001 .

In order to enhance efficiency of identification, an improved genetic algorithm (IGA) is proposed in this paper based on the original version. Additionally, all the modifications based on the original GA aim to achieve a better balance between efficiency and reliability. The procedure of IGA, mainly involving four aspects, is shown in Fig. 5.

\section{1) Parameters initialization}

Although the optimization results are less affected by the initial values in the GA, the closer to the optimal values the initial values are, the fewer iterations the optimization procedure costs. The population size is set to 100 . In contrast to the original GA initializing parameters completely at random, here the IGA performs initialization under the guidance of feasible region, making it more probable to approach optimal states. In particular, the feasible region of each parameter is divided into 10 parts. Then 10 samples are randomly selected in each part. This method can improve the probability of finding the range including the optimal solution at first generation.

2) Good individuals selection

The fitness function is inversion of the objective function in (5). So the parameter combination that diminish the objective function have larger fitness.

To avoid the loss of the optimal genes in the traditional roulette selection strategy. The $10 \%$ parameters with largest fitness are directly inherited to the next generation. 


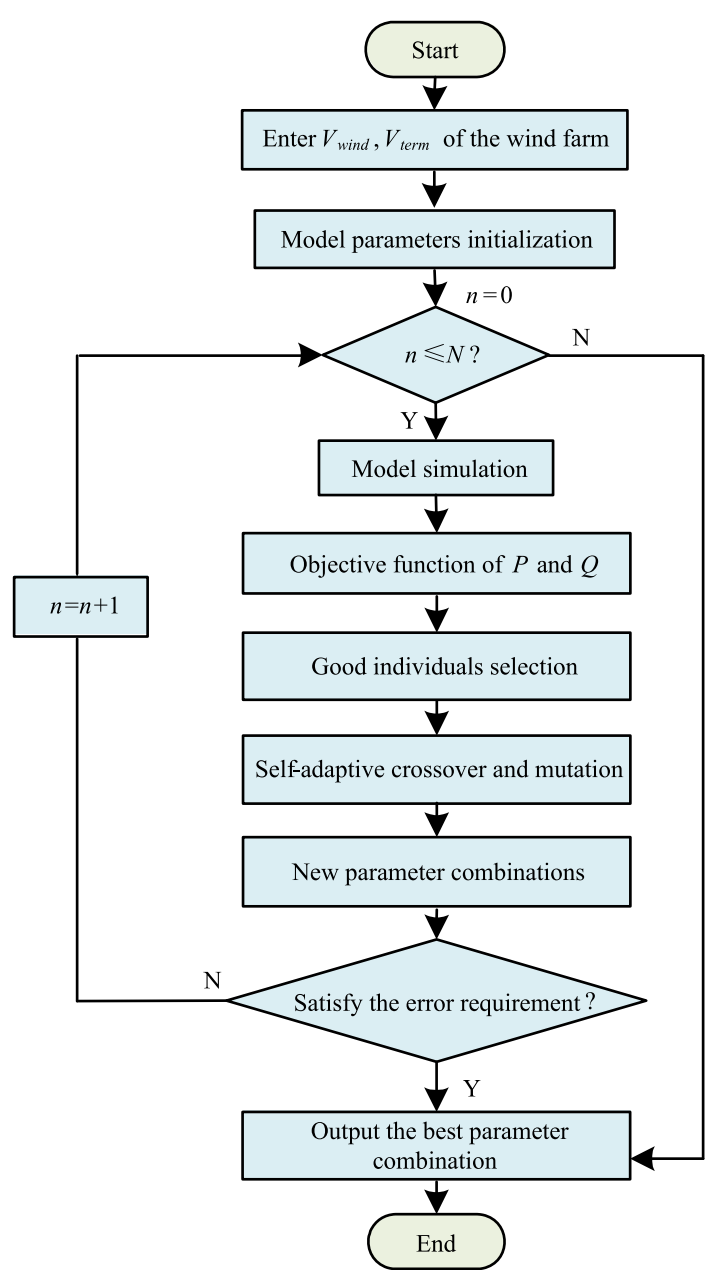

Fig. 5 Procedure of parameter identification based on IGA

The remaining $90 \%$ individuals are chosen by the roulette selection strategy.

\section{3) Self-adaptive crossover and mutation}

The individuals in a generation are randomly paired. And the crossover operation is carried out on the individuals in each pair to generate offspring. The crossover probability $P_{c}$ is used to control the frequency of crossover operation. A bigger $P_{c}$ can improve the search capability in the space, while a smaller $P_{c}$ can ensure the continuity of the search around the optimal value. So a self-adaptive technique is utilized in this paper, adjusting $P_{c}$ during the identification process by

$P_{c}^{n}=P_{c}^{n-1}+\left(0.5-P_{c}^{0}\right) / N$

where $n$ means the current number of iterations; $P_{c}^{n}$ is the crossover probability at $n$th iteration; $N$ is the maximum number of generations, which is 100 here; $P_{c}^{0}$ is the original value, which is set as 0.9 in this paper.
Multi-point mutation strategy is adopted in the mutation procedure. The purpose of mutation operation is to produce new individuals and expand the searching scope. Increasing the value of mutation probability $P_{m}$ can avoid falling into local optimum, while may destroy the optimum solution space. The recommended value range of $P_{m}$ is 0.001 to 0.1 . The $P_{m}$ is also adjusted in (7), according to the search results.

$$
\left\{\begin{array}{l}
P_{m}^{n}=P_{m}^{n-1}+\left(0.001-P_{m}^{0}\right) / N, \text { if } \tau=0 \\
P_{m}^{n}=P_{m}^{0}, \text { if } \tau=1
\end{array}\right.
$$

where $P_{m}^{n}$ is the mutation probability at $n$th iteration; $P_{m}^{0}$ is set as $0.1 ; \tau$ is the flag variable that decides whether to reset the $P_{m}^{n}$ as the original value. When the situation that incensement of the best fitness value of the current generation is less than $1 \%$ of the last generation occurs ten times continually, $\tau$ is equal to 1 . This means that the search range will change, if better values cannot be found after repeatedly search.

4) Iterative identification

The model simulation is incorporated in the IGA. Every new parameter combination created by the genetic manipulation is passed to the model simulation to calculate its object function value and fitness. Then good individuals are selected to be renew in a new round of genetic manipulation. The optimal parameter combination is obtained in the process of iterative identification.

The algorithm process will stop when the output error is in the range of the allowable error or the number of iterations reaches its maximum limit. In the latter situation, the optimum results, the optimal parameter combination in the last interaction probably is not the global optimum, considering the influence of crossover and mutation. So the best parameter combination must be selected from the optima in all generations.

\section{Simulation and verification}

\subsection{Model test based on the benchmark system}

A model simulation test is conducted on a simple system to verify the dynamic response of the model, as shown in Fig. 6. The test system is a benchmark system proposed by the WECC's Wind Generator Modeling Group [23]. There are 20 DFIGs that are rated at $1.5 \mathrm{MW}$ in the wind farm. The standard capacity of this system is 33 MVA. The power grid is represented by an infinite voltage source. The parameters of lines and transformers are given on per unit value (p.u).

Different forms of disturbances are imposed to test the response characteristics of the electrical control parts and 


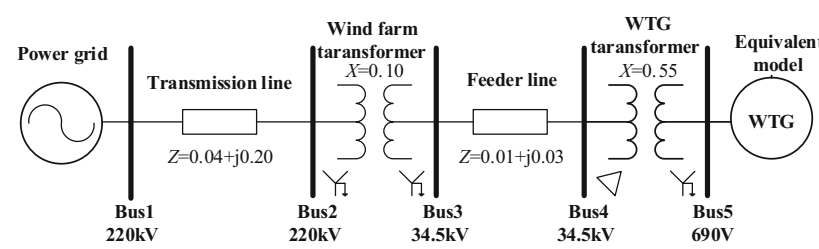

Fig. 6 Benchmark test system
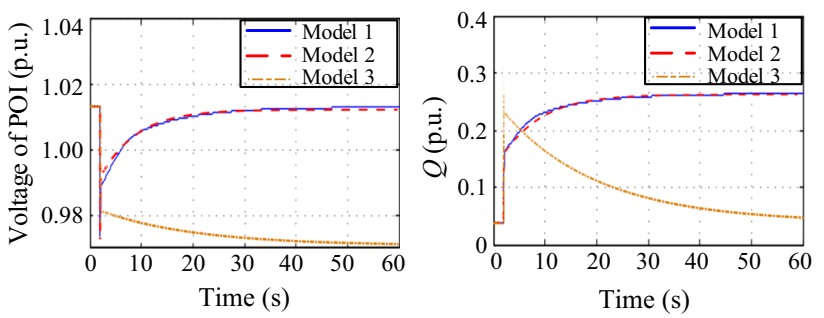

Fig. 7 Voltage and reactive power at Bus 2

the mechanical transmission parts. Several comparisons are made among the G.E. standard model (model 1), the equivalent model in this paper (model 2 ) and the traditional reduced-order model (model 3).

1) Test with grid voltage disturbance

A small step change of the grid voltage is carried out to test the control effects of reactive power and voltage control. The setting is that the voltage of grid drops from 1.05 p.u. to 0.97 p.u. at $1.0 \mathrm{~s}$. The voltage and the reactive power at Bus 2 (POI) are shown in Fig. 7.

The test results show that the equivalent model can timely increase reactive power to provide assistance for the voltage recovery, which is as same as the standard model, when the model is under constant-voltage control mode. While, the traditional model always uses constant reactive power control modes by setting the reactive power reference. So it's helpless for the voltage drop. It is obvious that the property of DFIGs is lost, although the model is simplified.

\section{2) Test on the wind speed disturbance}

The pitch angle control and regulation of active power are tested in this part by a wind ramp. The wind farm works under the rated condition. A wind ramp starts from $11.8 \mathrm{~m} /$ $\mathrm{s}$ at $10.0 \mathrm{~s}$, and reaches $17.8 \mathrm{~m} / \mathrm{s}$. Then, the wind speed remains steady for $30.0 \mathrm{~s}$. Its rise time and fall time are both $30.0 \mathrm{~s}$. The pitch angle and active power are shown in Fig. 8.

It is evident that the traditional reduced-order model that ignores the pitch angle control exhibits some undesirable characteristics in the period of wind disturbance. However, the equivalent model has almost the same features as the standard model, which can approximately maintain the
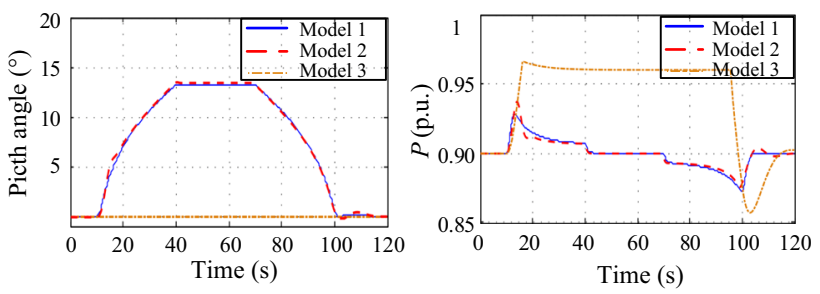

Fig. 8 Pitch angle and active power of the wind farm

output power approximate as a constant. The results demonstrate that the active control and pitch angle control are effective and more tally with the practical conditions.

\subsection{Validation of the identification algorithm}

The identification algorithm based on the IGA is verified on the CEPRI 36 bus system, as shown in Fig. 9. The wind farm containing $240 \times 1.5 \mathrm{MW}$ DFIGs is connected to the system at Bus8. A PMU is installed at the Bus31 to measure the dynamic information. The simulation data including amplitudes and phase angles of bus voltages, active power and reactive power outputs, are acquired to simulate PMU measurements. The standard capacity of this system is 100 MVA. Bus1 is treated as a slack point.

\section{1) Parameters selection and classification}

It is known that different dynamic modes may be stimulated by different disturbances [24]. In the model verification, it can be seen that the voltage and reactive power control may significantly affect the dynamic process when a transient disturbance occurs. Analogously, the same relationship exists between the pitch angle control and the wind disturbances. So the parameters may have different observability under different disturbances.

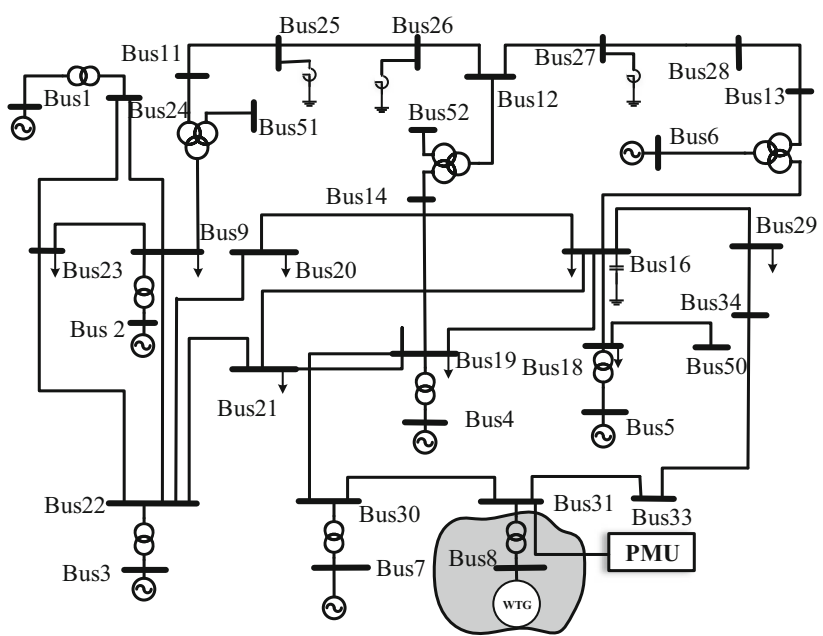

Fig. 9 CEPRI 36 bus system 
There will be two kinds of disturbances in this paper to classify and select the dominant parameters. One kind disturbance is a three-phase short-circuit fault at the Bus31 that lasts for $0.1 \mathrm{~s}$ (Case 1). The other kind disturbance is a gust that starts at $2.0 \mathrm{~s}$ with an initial speed of $14 \mathrm{~m} / \mathrm{s}$ and lasts for $20 \mathrm{~s}$. The maximum of the speed is $20 \mathrm{~m} / \mathrm{s}$ (Case 2). The parameters' trajectory sensitivity curves under both kinds of disturbances are given in Fig. A2 and Fig. A3 in Appendix A. The parameters of PI controllers are assumed to be similar to individual wind turbines. And they are also less affected by the wind farms' operating conditions, service life, etc. So they are preset and regarded as unchanged. Only the parameters that are sensitive and variable, such as $X_{e q}, E_{I Q \max }, E_{I Q \min }, T_{g}, T_{w t}, K_{t g}$ and $D_{t g}$ are analyzed in this paper. The parameters $X_{e q}, E_{I Q \max }$ and $E_{I Q \min }$ that are more associated with electrical response characteristics are referred to as electrical parameters. Meanwhile, the parameters $T_{g}, T_{w t}, K_{t g}$ and $D_{t g}$ are referred to as mechanical parameters.

From the results, it is found that those parameters are uncoupled to each other except that some parameters' sensitivities are too small to identify. In order to compare the identifiability of those parameters, the average sensitivities are listed in Table 1 . Every number in the table represents the corresponding sensitivity with respect to $P$ or $Q$.

It indicates that the parameters playing an important role at the voltage and reactive power controls such as $X_{e q}$ and $E_{I Q \max }$ are easier to be identified under the transient fault. The parameters such as $T_{g}, T_{w t}$ and $K_{t g}$ have some identifiable degrees in both cases. But they are relatively more sensitive under the gust disturbance. However, it is difficult to identify $E_{I Q \min }$ and $D_{t g}$ in either case. Therefore, $X_{e q}$ and $E_{I Q \max }$ are identified under the transient fault, while $T_{g}, T_{w t}$ and $K_{t g}$ are under the gust disturbance.

\section{2) Parameter identification}

The parameter identification method can play an effective role, when there is a disturbance in the wind farm operation. Different parameters are identified under different situations. The simulation experiment is also in the CEPRI 36 bus system with the same disturbances in the Case 1 and Case 2.

The POI bus is probably not equipped with PMU, just as this case in the Fig. 9. If the resistance and ratio of the

Table 1 Average sensitivities of the parameters

\begin{tabular}{lllllllll}
\hline Case & & $X_{e q}$ & $E_{I Q \max }$ & $E_{I Q \min }$ & $T_{g}$ & $T_{w t}$ & $K_{t g}$ & $D_{t g}$ \\
\hline Case 1 & $P$ & 0.363 & 0.441 & 0.006 & 0.077 & 0.049 & 0.016 & 0.001 \\
& $Q$ & 0.101 & 0.011 & 0.003 & 0.019 & 0.006 & 0.003 & 0.000 \\
Case 2 & $P$ & 0.001 & 0.000 & 0.000 & 0.088 & 0.140 & 0.034 & 0.000 \\
& $Q$ & 0.009 & 0.000 & 0.000 & 0.165 & 0.261 & 0.064 & 0.000 \\
\hline
\end{tabular}

Table 2 Comparisons of parameter identification results

\begin{tabular}{llllll}
\hline Parameter & $X_{e q}$ & $E_{I Q \max }$ & $T_{g}$ & $T_{w t}$ & $K_{t g}$ \\
\hline Actual value & 0.500 & 0.400 & 1.500 & 8.600 & 1.420 \\
Identified value & 0.489 & 0.387 & 1.519 & 8.608 & 1.437 \\
Relative error & $2.20 \%$ & $3.25 \%$ & $1.27 \%$ & $0.12 \%$ & $1.20 \%$ \\
\hline
\end{tabular}

transformer between bus 8 and bus 31 is known, the information of the wind farm can be calculated from the PMU measurements by some state estimation methods.

The identified values of those parameters based on IGA are compared with the actual values in Table 2. It is obvious that the identified values are rather close to the actual values. The relative errors are all within the range of the errors permitted. The results verify the effectiveness of the method.

Furthermore, the identification results are shown in Fig. 10 and Fig. 11, where the response results of measured data, identified data and the outputs based on empirical parameters are comprehensively compared.

The comparisons show that the response based on identification is in general agreement with the practical measurement results, whereas the response based on empirical parameters are greatly different. It further suggests that power system security analysis using empirical parameters of wind farm models is likely to deviate from the actual situation. Moreover, it reveals the parameter identified from measurement data of WAMS is of desirable reliability.
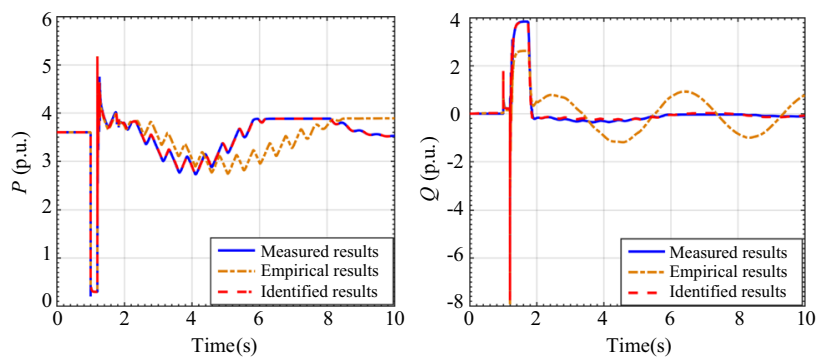

Fig. 10 Comparisons of responses under the transient fault
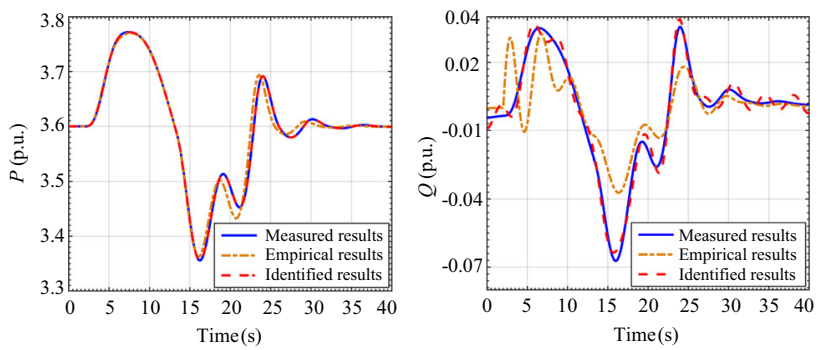

Fig. 11 Comparisons of responses under the gust disturbance 

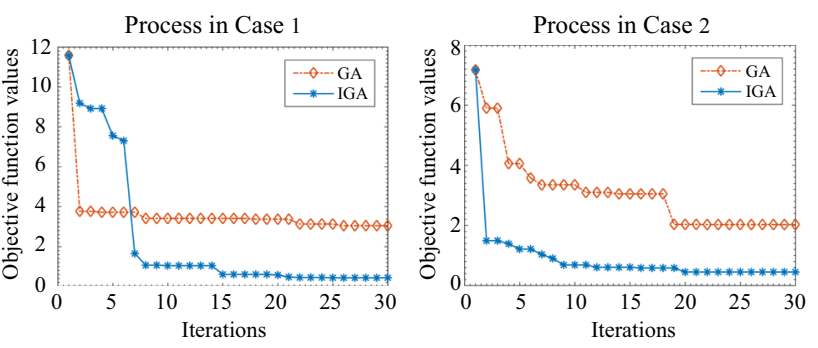

Fig. 12 Comparisons between the GA and IGA

Table 3 Identified values of parameters and fitting degrees

\begin{tabular}{lrrrrr}
\hline Number & 1 & \multicolumn{1}{l}{2} & 3 & \multicolumn{1}{l}{4} & \multicolumn{1}{l}{5} \\
\hline$X_{\text {eq }}$ & 0.489 & 0.491 & 0.486 & 0.498 & 0.501 \\
$E_{I Q \min }$ & -0.481 & -0.205 & -0.303 & -0.428 & -0.187 \\
Fitting degree of $P$ & $98.7 \%$ & $98.9 \%$ & $98.6 \%$ & $99.4 \%$ & $99.2 \%$ \\
\hline
\end{tabular}

To validate the advantages of the IGA related to the traditional GA, the iterative process of the two kinds algorithms in both identification cases are shown in the Fig. 12, where the objective function values are given at same iterations.

It is shown that the convergence rate of the errors is greatly improved in the IGA with the adaptive crossover operator and mutation operator. Therefore, the IGA proposed in this paper is effective to enhance the global search capability of genetic algorithm during the procedure of both identification cases.

To prove that the sensitivity is a good index for the identifiability, the parameters with small sensitivities, such as $E_{I Q \min }$ and $D_{t g}$, were added into the parameter set for identification. The fitting degrees of active power and identified results of $X_{e q}$ and $E_{I Q \min }$ in five experiments are listed in Table 3.

In some high fitting degree situations, the values of $X_{e q}$ are relative steady, while the values of $E_{I Q \min }$ are randomly distributed in its feasible regions, which shows that those parameters with small sensitivities are difficult to be accurately identified. So they are usually specified as empirical values. It also implies that the parameters selection based on sensitivity can effectively remove those intractable parameters and improve the identification efficiency.

\section{3) Parameter validation}

However, there may exist several sets of parameters that produce similar responses from the model. So the parameters identified by the IGA need to be further selected by the responses under stochastic wind that frequently appears in practice (Case 3 ).

To validate the effectiveness of the optimized model parameters, a new set of parameters (Set B, Table 4)
Table 4 Values of parameter Set B

\begin{tabular}{llllll}
\hline Parameter & $X_{e q}$ & $E_{I Q \max }$ & $T_{g}$ & $T_{w t}$ & $K_{t g}$ \\
\hline Set B & 0.779 & 0.317 & 1.676 & 7.602 & 1.358 \\
\hline
\end{tabular}
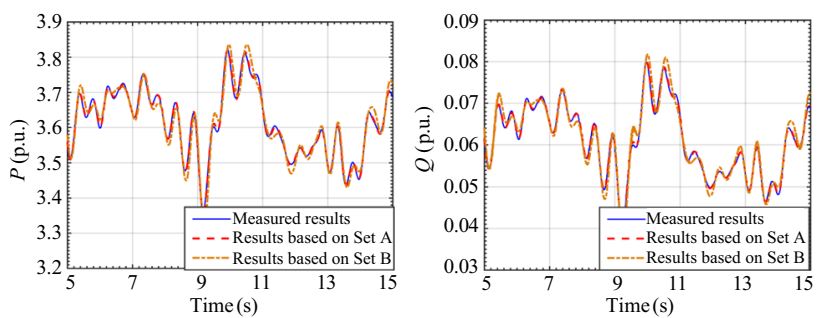

Fig. 13 Comparisons of responses based on Set A and Set B in Case 3

producing comparable responses as the previously identified parameters (Set A, see Table 2) is obtained from identification processes by expanding the feasible regions of parameters, as listed in Table A1 in Appendix A.

The measured results and the responses based on the parameter Set A and Set B are compared in Fig. 13.

The fitting degree of active power based on the parameters of Set A is $98.55 \%$, while the counterpart based on Set B is $92.15 \%$. Meanwhile, the fitting degrees of reactive power corresponding to Set A and B are $97.32 \%$ and $91.08 \%$, respectively. It is obvious that the results based on parameter Set A stay much closer to the practical measurements than the parameter Set B in Case 3. So the parameters of Set A are selected as the optimal parameters. Such a verification also reveals that the optimal parameters should be well adaptive under other disturbance occasions.

\section{Conclusion}

In this paper, a wind farm modeling and parameter identification approach based on measured data of PMUs is proposed to solve the problem of unreliable models and the inaccurate parameters. An equivalent wind farm model involving basic structural characters and control principles is built to effectively simulate the actual response of the wind farm. Dominant parameters are selected and classified by trajectory sensitivity analysis under two kinds of disturbances, which helps to tackle the intricate and laborious identification issue of multiple parameters. Besides, an improved generic algorithm, enhancing the capability of globally and efficiently searching optimal parameters, is developed. In particular, the genetic manipulation is optimized with the self-adaptive cross and selection operators, attempting to find a balance between efficiency and reliability. 
The test on the WECC benchmark system under voltage steps and wind ramps demonstrates that the response of this model is coincided with the standard model, which can reflect the real dynamic characteristics better than the traditional model. In addition, the validation of the identification algorithm is carried out on the CEPRI 36-bus system, which suggests the model response with identified parameters based on WAMS are more accurate and reasonable than with the empirical parameters. Then the IGA is compared with the traditional GA. which validates the better global search capability of this method. Finally, the optimal parameters are selected and validated under the stochastic wind condition in case of multi-solution situations.

This paper has provided a reliable modeling method based on WAMS for wind farms. In relevant future work, further validations using field data will be necessary to demonstrate the effectiveness in the real system.
Additionally, the modeling method can be also employed to assist power system analysis and control strategies with large-scale wind power integrated in the future.

Acknowledgments This work was supported by National Basic Research Program of China (No. 2012CB215206) and National Natural Science Foundation of China (No. 51107061).

Open Access This article is distributed under the terms of the Creative Commons Attribution 4.0 International License (http:// creativecommons.org/licenses/by/4.0/), which permits unrestricted use, distribution, and reproduction in any medium, provided you give appropriate credit to the original author(s) and the source, provide a link to the Creative Commons license, and indicate if changes were made.

\section{Appendix A}

See Tables A1, A2 and Figs. A1, A2 and A3.

Table A1 Model parameters adopted by wind farms in a regional power network simulation

\begin{tabular}{lllll}
\hline Area & Area 1 & Area 2 & Area 3 & Area 4 \\
\hline Total number of models & 34 & 72 & 94 & 151 \\
Number of models with unified parameters & 33 & 72 & 89 & 125 \\
\hline
\end{tabular}

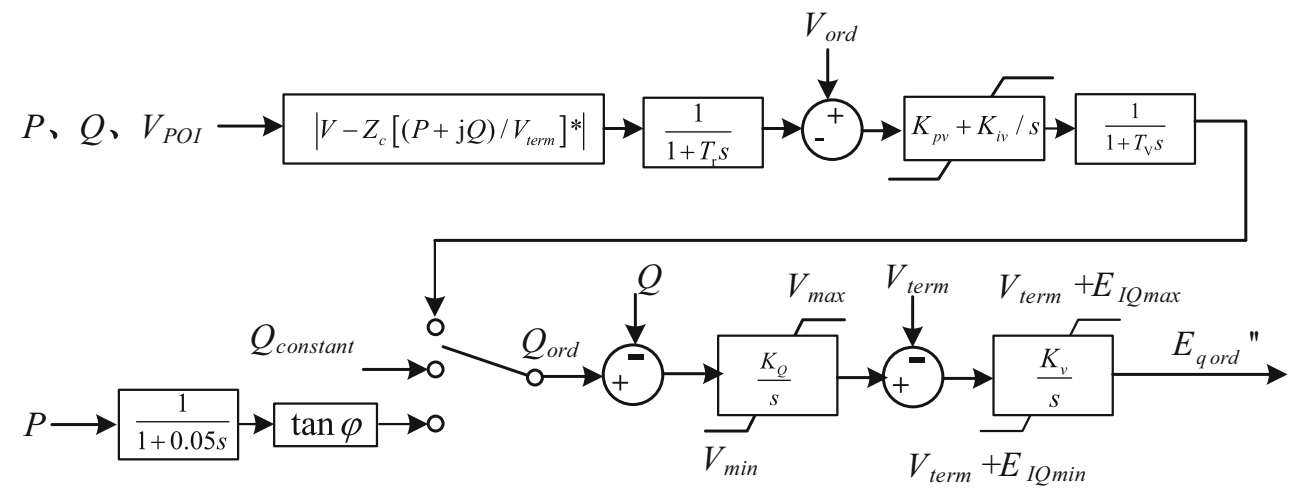

Fig. A1 Reactive control module

Table A2 Parameters of the wind farm model and their common empirical values in p.u

\begin{tabular}{llll}
\hline Equivalent reactance $\left(X_{e q}\right)$ & 0.55 & Pitch angle PI control $\left(K_{p p}\right)$ & 150 \\
Integral coefficient of $Q\left(K_{Q i}\right)$ & 0.05 & Pitch angle PI control $\left(K_{p i}\right)$ & 25.0 \\
Integral coefficient of $V\left(K_{V i}\right)$ & 30.0 & Inertia coefficient of generator $\left(T_{g}\right)$ & 1.14 \\
Upper bound of $E_{q}^{\prime \prime}$ change $\left(E_{I Q \max }\right)$ & 0.30 & Inertia coefficient of turbine $\left(T_{w t}\right)$ & 7.64 \\
Lower bound of $E_{q}^{\prime \prime}$ change $\left(E_{I Q \min }\right)$ & -0.35 & Stiffness coefficient of shaft $\left(K_{t g}\right)$ & 1.25 \\
Control mode of $Q\left(Q_{f l a g}\right)$ & Constant-voltage & Damping coefficient of shaft $\left(D_{t g}\right)$ & 1.50 \\
\hline
\end{tabular}



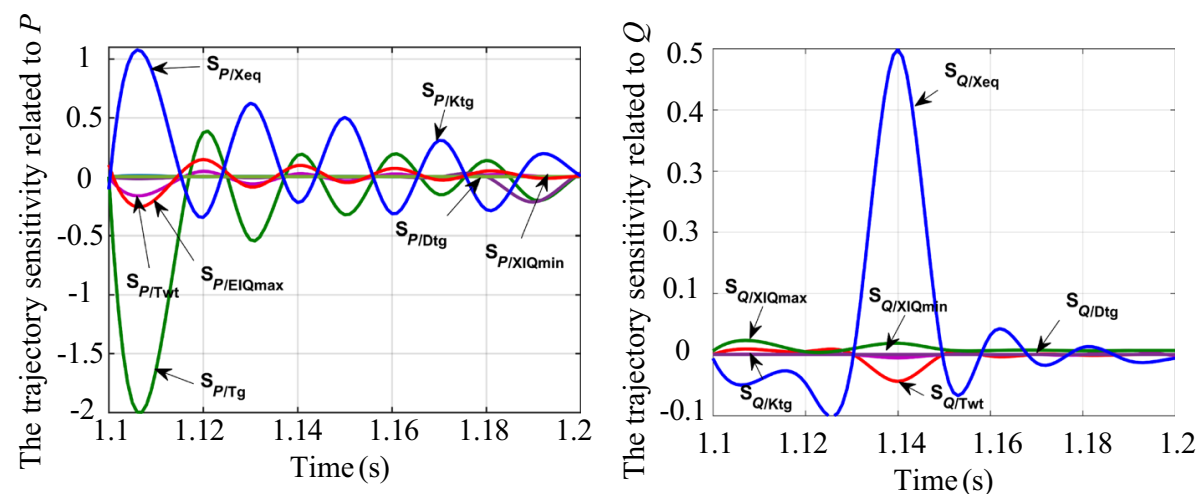

Fig. A2 Trajectory sensitivity curves of the parameters under the transient fault
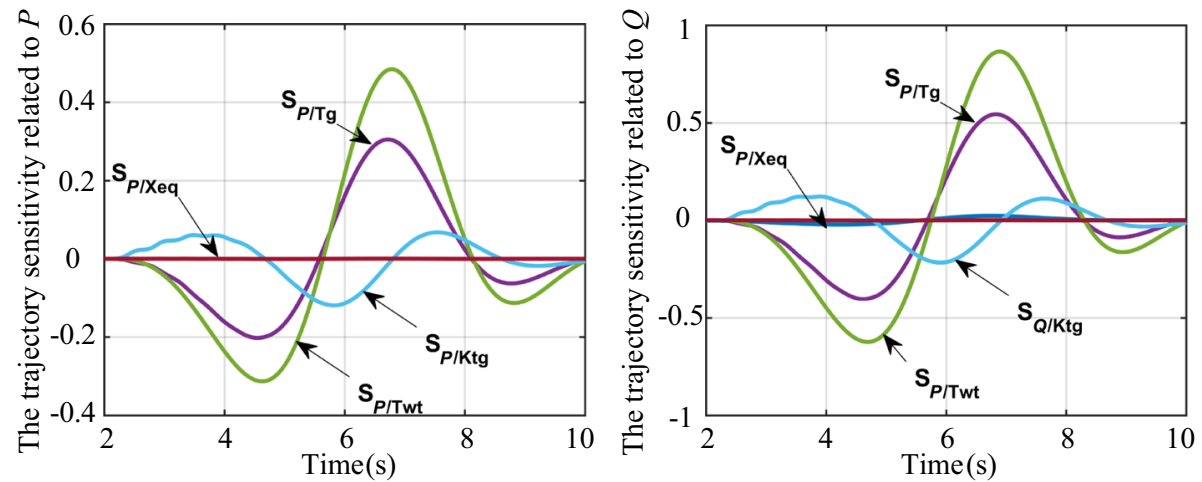

Fig. A3 Trajectory sensitivity curves of the parameters under the gust disturbance

\section{References}

[1] Usaola J, Ledesma P, Rodriguez JM et al (2003) Transient stability studies in grids with great wind power penetration. Modelling issues and operation requirements. In: Proceedings of the 2003 IEEE Power Engineering Society General Meeting, Toronto, Ont., Canada, 13-17 July 2003, pp 1534-1541

[2] Shi LB, Sun SM, Yao LZ et al (2014) Effects of wind generation intermittency and volatility on power system transient stability. IET Renew Power Gener 8(5):509-521

[3] Sun YS, Tang XS (2014) Cascading failure analysis of power flow on wind power based on complex network theory. J Mod Power Syst Clean Energy 2(4):411-421. doi:10.1007/s40565014-0088-5

[4] Chowdhury MA, Shen WX, Hosseinzadeh N et al (2015) Transient stability of power system integrated with doubly fed induction generator wind farms. IET Renew Power Gener 9(2):184-194

[5] Pan X, Liu Z, Liu WX et al (2015) Wind farm polymerization influences on security and economic operation in power system based on Copula function. J Mod Power Syst Clean Energy 3(3):381-392. doi:10.1007/s40565-015-0147-6

[6] Liu HK, Xie XR, Li Y et al (2015) A small-signal impedance method for analyzing the SSR of series-compensated DFIGbased wind farms. In: Proceedings of the 2015 IEEE Power and Energy Society General Meeting, Denver, CO, USA. 26-30 July 2015, 5 pp
[7] Cheng L, Liu MJ, Sun YZ et al (2013) A multi-state model for wind farms considering operational outage probability. J Mod Power Syst Clean Energy 1(2):177-185. doi:10.1007/s40565013-0025-z

[8] Ali M, Ilie IS, Milanovic JV et al (2013) Wind farm model aggregation using probabilistic clustering. IEEE Trans Power Syst 28(1):309-316

[9] Pan XP, Zhang C, Ju P et al (2015) Coherency-based dynamic equivalence of wind farm composed of doubly fed induction generators. Power Syst Technol 39(3):621-627

[10] Zou JX, Peng C, Xu HB et al (2015) A fuzzy clustering algorithm-based dynamic equivalent modeling method for wind farm with DFIG. IEEE Trans Energy Convers 30(4):1329-1337

[11] Brochu J, Larose C, Gagnon R (2010) Validation of Single- and multiple-machine equivalents for modeling wind. Power Plants IEEE Trans Energy Convers 26(2):532-541

[12] Xue F, Song XF, Chang K et la (2013). Equivalent modeling of DFIG based wind farm using equivalent maximum power curve. In: Proceedings of the IEEE Power and Energy Society General Meeting (PES), Vancouver, BC, 21-25 July 2013, 5 pp

[13] Zhang YC, Muljadi E, Kosterev D et al (2014) Wind power plant model validation using synchrophasor measurements at the point of interconnection. IEEE Trans Sustain Energy 6(3):984-992

[14] Feijóo A, Cidrás J, Carrillo C (2000) A third order model for the doubly-fed induction machine. Electric Power Syst Res 56(2): 121 
[15] Elkington K, Ghandhari M (2009) Comparison of reduced order doubly fed induction generator models for nonlinear analysis. In: Proceedings of the 2009 IEEE Electrical Power and Energy Conference, Montreal, QC, 22-23 October 2009, 6 pp

[16] Muljadi E, Ellis A (2008). Validation of wind power plant models. In: Proceedings of the 2008 IEEE Power and Energy Society General Meeting-Conversion and Delivery of Electrical Energy in the 21st Century, Pittsburgh, PA. 20-24 July 2008, $7 \mathrm{pp}$

[17] Tsai C, Lee W, Nashawati E et al (2012) PMU based generator parameter identification to improve the system planning and operation. In: Proceedings of the 2012 IEEE Power and Energy Society General Meeting, San Diego, CA. 22-26 July 2012, 8 pp

[18] Wang B, Lu JJ (2011) A method for online analyzing excitation systems performance based on PMU measurements. In: Proceedings of the 2011 International Conference on Advanced Power System Automation and Protection (APAP), Beijing, China, 16-20 October 2011, pp 1538-1542

[19] Zhang XR, Lu C, Han Y et al (2015) Ambient signal based load model parameter identification using optimization method. In: Proceedings of the 2015 IEEE Power and Energy Society General Meeting, Denver, CO. 26-30 July 2015, 5 pp

[20] Miller NW, Price WW, Sanchez-Gasca JJ (2004) Modeling of GE wind turbine-generators for grid studies. version 3.3. GE International, Inc, Schenectady, NY

[21] Pan XP, Gao Y, Jin YQ et al (2013) Parameter identification of drive system for fixed-speed wind power generation units. Power Syst Technol 37(7):1990-1994

[22] Ju P, Guo L, Gao CP et al (2010) Frequency-domain sensitivities with application to power system modeling. Proc CSEE 30(28):19-24

[23] Price WW, Sanchez-Gasca JJ (2006) Simplified wind turbine generator aerodynamic models for transient stability studies. In: Proceedings of the 2006 IEEE PES Power Systems Conference and Exposition, Atlanta, GA. October 29-November 1 2006, pp 986-992
[24] Pan XP, Ju P, Qian X et al (2013) A two-step method for estimating dfig parameters in a wind turbine and the measurement selection. Proc CSEE 33(13):116-126

Yinfeng WANG received the B.S. degree from Tsinghua University in 2014. From 2014, he started to pursue his Ph.D. degree at Tsinghua University, Beijing, China. His current research focuses on the modeling and identification of wind farms based on WAMs.

Chao LU received the B.S. and Ph.D. degrees in electrical engineering from Tsinghua University, Beijing, China, in 1999 and 2005, respectively. He is currently an Associate Professor with the Department of Electrical Engineering, Tsinghua University. His research interests include power system analysis and control, and intelligent control applications.

Lipeng ZHU received the B.S. degree from Huazhong University of Science and Technology, Wuhan, China, in 2012, and the M.S. degree from Wuhan University, Wuhan, China, in 2015, both in electrical engineering. Currently he is working towards the Ph.D. degree at Tsinghua University, Beijing, China. His research mainly deals with power system voltage stability analysis based on data mining.

Guoli ZHANG is a senior electrical engineer of the Power Research Institute, Eastern Inner Mongolia Power Grid of China. His research interests include power system analysis and control.

Xiu LI is an electrical engineer of the Power Research Institute, Eastern Inner Mongolia Power Grid of China. His research interests include power system modeling and simulation.

Ying CHEN is an electrical engineer of the Power Research Institute, Eastern Inner Mongolia Power Grid of China. Her research interests include wind power generation control. 